\title{
Metabolic syndrome, its components and mortality: A population-based study
}

\author{
Saeideh Mazloomzadeh*1, Fatemeh Karami Zarandi ${ }^{1}$, Alireza Shoghli ${ }^{2}$, Hossain Dinmohammadi
}

\section{Abstract}

Background: The association between Metabolic syndrome (MetS), its components and mortality has not been clearly established. The aim of this study was to determine the effects of Mets and its components on all cause and cause-specific mortality and to examine whether MetS or its components were better predictors of mortality.

Methods: In this retrospective cohort study, we used data from the Zanjan Healthy Heart Study performed in 2003 on 4000 persons. Based on the definitions provided by the NCEP- ATPIII, 1051 participants with MetS and 1219 with none or one of its components at study entry were enrolled. Information regarding the mortality and morbidity of 502 participants with MetS and 523 controls was collected in 2013 by telephone. Cause of death was defined as Cardio-Vascular Disease (CVD) or non-CVD. Data were analyzed using the Cox Proportional Hazards model to estimate the hazard ratios predicted by MetS and its individual components.

Results: The median duration of follow-up was $104 \pm 10.7$ months. Thirty-five deaths occurred, including 18 cardiovascular deaths. The proportion of those with CVD, hypertension, diabetes or hospital stay was statistically higher in MetS patients than controls $(\mathrm{p}<0.0001)$. The hazard ratios of all-cause and cardiovascular mortality for those with MetS were 1.75 (\%95CI: $0.88-3.47)$ and 3.66 (\%95CI: 1.2-11.1) higher than controls, respectively. Among the components of MetS, only hypertension predicted a higher risk of allcause and CVD mortality after adjusting for age and sex.

Conclusion: The results of this study indicated that MetS was associated with a higher risk of CVD mortality, morbidity, and hospital stay. Among the components of MetS, the association of hypertension was stronger compared to MetS as a whole. Therefore, this study confirms that MetS is a risk factor for CVD mortality, but not beyond the risk associated with its individual components.

Keywords: Metabolic Syndrome, Mortality, Hypertensionn

Conflicts of Interest: None declared

Funding: Zanjan University of Medical Sciences and Zanjan Metabolic Diseases Research Center

\section{*This work has been published under CC BY-NC-SA 1.0 license. \\ Copyright $\odot$ Iran University of Medical Sciences}

Cite this article as: Mazloomzadeh S, Karami Zarandi F, Shoghli A, Dinmohammadi H. Metabolic syndrome, its components and mortality: A population-based study. Med J Islam Repub Iran. 2019 (27 Feb);33:11. https://doi.org/10.47176/mjiri.33.11

\section{Introduction}

Metabolic syndrome refers to a cluster of classic cardiovascular risk factors such as hypertension, obesity, glucose intolerance and dyslipidemia. A number of studies have examined the association between metabolic syndrome and all-cause or CVD mortality around the world. Some studies have reported that MetS is related to an increase of all-cause mortality (1-5), CVD mortality (6-8),

Corresponding author: Dr Saeideh Mazloomzadeh, smazloomzadeh@zums.ac.ir

1. Zanjan Metabolic Diseases Research Center, Zanjan University of Medical Sciences, Zanjan, Iran

2. Social Determinants of Health Research Center, Zanjan University of Medical Sciences, Zanjan, Iran

3. Molecular Medicine Department, Faculty of Medicine, Zanjan University of Medical Sciences, Zanjan, Iran or both $(9,10)$, however, other studies have found no significant association (11-14).

Studies investigating the association between metabolic syndrome and mortality were mostly conducted within the elderly population in western nations while information regarding Asian populations, particularly Iran, is scarcer $(15,16)$. To our knowledge, only one study,

\section{$\uparrow$ What is "already known” in this topic:}

The effect of metabolic syndrome on all-cause or

CVD mortality has been examined within the elderly population in western countries. However, Information on the association between MetS, its components and mortality has not been clearly established particularly in Asian populations including Iran.

\section{$\rightarrow$ What this article adds:}

This study indicated that MetS is a risk factor for CVD mortality, but not beyond the risk associated with its individual components. 
Isfahan Cohort Study in Iran assessed the association between MetS and incidence of ischemic heart disease (IHD) during a 5 year follow-up period and reported that metabolic syndrome was a major determinant of IHD in the middle-aged population (17). However, the association between MetS and all-cause and CVD mortality was not evaluated in this region.

Since cardiovascular events are the most common cause of death in most countries including ours and MetS is a cluster of cardio-metabolic abnormalities which are related to poor cardiovascular outcomes, it is important to investigate that having MetS is a valuable clinical means to distinguish those with an increased risk of CVD. The association between metabolic syndrome and CVD mortality varies by population based on ethnicity, cultural and lifestyle factors. Therefore, due to a high prevalence of MetS in Iran and rare data on this association, we performed this study to evaluate the association between MetS and all-cause and CVD mortality in this region.

Given that the definition of MetS requires the presence of at least three of five components, another important issue is whether MetS successfully predicts cardiovascular death to a higher degree than its individual components. The results of a systematic review indicated that more research is necessary in order to investigate if the prognostic significance of metabolic syndrome outweighs the risk linked with the sum of its separate components (9). Thus, in this study, we also examined whether MetS better predicted mortality in comparison to its components.

\section{Methods \\ Baseline}

This retrospective cohort study was performed using data from the Zanjan Healthy Heart Study. Data gained from the study and laboratory methods have been described in detail previously (18). A brief summary is that the study was conducted in Zanjan, north-west of Iran, in 2003. About 4000 subjects older than 15 years were selected from the general population by a multistage cluster sampling. Inclusion criteria for both groups was participation in the Zanjan Healthy Heart Study in 2003 and being available at the time of study, for exposed group was having MetS at study entry based on the definitions provided by the National Cholesterol Education Program-Adult Treatment Panel III (NCEP- ATPIII) and for non-exposed group was having none or one of the MetS components. Those who had between two and four of the MetS components according to the NCEP- ATPIII, were excluded.

Information on demographic factors, medical history, and lifestyle risk factors was collected at baseline through a standardized questionnaire. Physical examinations and measurements were carried out by trained nurses and physicians. Blood samples were obtained in the morning from individuals after 12 hours of fasting. Written informed consent was acquired from all participants. The study was approved by the Ethics Committees of Zanjan University of Medical Sciences.

\section{Follow-up}

Data from the Zanjan Healthy Heart Study showed that at baseline there were 1051 patients with metabolic syndrome based on NCEP (ATPIII) criteria and 1219 subjects who had no or one of the components of metabolic syndrome. Exposure was defined as having metabolic syndrome and the outcome was death. Data on study outcomes were collected in 2013 by telephone. Participants were asked to report any major disease (such as cancer, cardiovascular events, diabetes, hypertension, pulmonary disease, liver or kidney disease, peptic ulcer) or hospital stays. Information on subject mortality (date and cause of death) was collected from his/her family. Cause of death was defined as either CVD (such as related to coronary heart disease, cerebrovascular disease, and heart failure) or non-CVD (due to cancer, accident, etc.). Using these methods, mortality follow-up was complete for $45 \%$ of participants. Thus, this study was conducted on 502 patients with metabolic syndrome (exposed group) and 523 healthy individuals (unexposed group). The sample size was calculated to be at least 483 for each group based on the following information: $\mathrm{P}_{1}=0.53$ (the proportion of death in those with MetS), $\mathrm{P}_{2}=0.44$ (the proportion of death in those without MetS), $\alpha=0.05,1-\beta=0.80$.

\section{Definition of metabolic syndrome}

Metabolic syndrome was defined based on NCEP (ATPIII) criteria which included five components: (1) central obesity (waist circumference $\geq 102 \mathrm{~cm}$ in men and $\geq 88 \mathrm{~cm}$ in women, (2) elevated triglyceride level $(\geq 150$ $\mathrm{mg} / \mathrm{dL}$ ), (3) low HDL-C level (men: $<40 \mathrm{mg} / \mathrm{dL}$, women: $<50 \mathrm{mg} / \mathrm{dL}$ ), (4) hypertension (systolic BP: $\geq 130 \mathrm{~mm} \mathrm{Hg}$, diastolic BP: $\geq 85 \mathrm{~mm} \mathrm{Hg}$, or treated hypertension), or elevated fasting glucose level (EFG) (glucose, $\geq 100$ $\mathrm{mg} / \mathrm{dL}$ or treated diabetes). Metabolic syndrome was considered as having at least three of these five components.

\section{Statistical analyses}

The Kolmogorov-Smirnov test was used to assess the distribution of quantitative variables. Values were expressed as numbers (percentages), and mean \pm standard deviation, as appropriate. Comparisons were achieved via separate methods, for categorical variables the chi-square test, for normally distributed variables the independent ttest, and for non-normally distributed variables the MannWhitney test was utilized. A Cox proportional hazards model was created to examine the association between mortality and the relevant variables. All statistical analyses were performed using the SPSS 16.0 and a p-value less than 0.05 was considered as statistically significant.

\section{Results}

The baseline characteristics of study participants with metabolic syndrome are detailed in Table 1 . The mean age of the MetS patients was higher than controls $(\mathrm{p}<0.0001)$ and $283(56.4 \%)$ of MetS patients and $233(44.6 \%)$ of the controls were female $(\mathrm{p}<0.0001)$. Subjects with MetS had a higher Body Mass Index (BMI), waist circumference, fasting plasma glucose, systolic or diastolic blood pressure, total cholesterol, triglyceride levels, LDL-C levels and lower HDL-C levels in comparison with those without MetS (Table 1). 


\begin{tabular}{|c|c|c|c|c|}
\hline \multicolumn{2}{|l|}{ Characteristics } & $\begin{array}{c}\text { With MetS } \\
(\text { Mean } \pm \text { SD) }\end{array}$ & $\begin{array}{c}\text { Without MetS } \\
(\text { Mean } \pm \text { SD })\end{array}$ & $\mathrm{p}$ \\
\hline \multicolumn{2}{|c|}{ Age (years) } & $44.6 \pm 16.46$ & $31.17 \pm 14.48$ & $<0.0001$ \\
\hline \multicolumn{2}{|c|}{ Body Mass Index $(\mathrm{kg} / \mathrm{m} 2)$} & $27.78 \pm 4.72$ & $22.08 \pm 3.77$ & $<0.0001$ \\
\hline \multicolumn{2}{|c|}{ Waist circumference $(\mathrm{cm})$} & $93.73 \pm 11.46$ & $77.73 \pm 10.18$ & $<0.0001$ \\
\hline \multicolumn{2}{|c|}{ Systolic blood pressure (mmHg ) } & $131.29 \pm 23.07$ & $109.18 \pm 14.61$ & $<0.0001$ \\
\hline \multicolumn{2}{|c|}{ Diastolic blood pressure (mmHg) } & $82.8 \pm 11.96$ & $70.65 \pm 8.98$ & $<0.0001$ \\
\hline \multicolumn{2}{|c|}{ Fasting glucose $(\mathrm{mg} / \mathrm{dl})$} & $101.84 \pm 40.1$ & $31.17 \pm 14.48$ & $<0.0001$ \\
\hline \multicolumn{2}{|c|}{ Cholesterol(mg/dl) } & $206.75 \pm 42.82$ & $22.08 \pm 3.77$ & $<0.0001$ \\
\hline \multicolumn{2}{|c|}{ Triglyceride(mg/dl) } & $228.22 \pm 126.18$ & $77.73 \pm 10.18$ & $<0.0001$ \\
\hline \multicolumn{2}{|c|}{$\operatorname{HDL}(\mathrm{mg} / \mathrm{dl})$} & $36.73 \pm 5.96$ & $109.18 \pm 14.61$ & $<0.0001$ \\
\hline \multicolumn{2}{|l|}{$\mathrm{LDL}(\mathrm{mg} / \mathrm{dl})$} & $129.57 \pm 40.11$ & $70.65 \pm 8.98$ & $<0.0001$ \\
\hline \multicolumn{2}{|c|}{ Variable } & $\begin{array}{c}\text { With MetS } \\
\text { N }(\%)\end{array}$ & $\begin{array}{c}\text { Without MetS } \\
\text { N }(\%)\end{array}$ & $\mathrm{p}$ \\
\hline \multirow[t]{2}{*}{ Mortality } & No & $480(\% 95.6)$ & $510(\% 97.5)$ & 0.095 \\
\hline & Yes & $22(\% 4.4)$ & $13(\% 2.5)$ & \\
\hline \multirow[t]{2}{*}{ Hospitalization } & No & $228(\% 45.4)$ & $357(\% 68.3)$ & $<0.0001$ \\
\hline & Yes & $274(\% 54.6)$ & $166(\% 31.7)$ & \\
\hline \multirow[t]{4}{*}{ Diseases } & No & $273(\% 54.4)$ & $478(\% 91.4)$ & $<0.0001$ \\
\hline & ${ }^{\mathrm{a}} \mathrm{CVD}$ & $16(\% 3.2)$ & $5(\% 1.0)$ & \\
\hline & ${ }^{\mathrm{a} D M} / \mathrm{HTN}$ & $110(\% 21.9)$ & $23(\% 4.4)$ & \\
\hline & Others & $103(\% 20.5)$ & $17(\% 3.3)$ & \\
\hline
\end{tabular}

${ }^{\mathrm{a}} \mathrm{CVD}=$ Cardio-Vascular Diseases, $\mathrm{DM}=$ Diabetes Melitus, HTN= Hypertension.

The mean duration of follow-up was $104 \pm 10.7$ months, and its median was 107.1 months (range: 1 to 108.5 months). Thirty-five deaths occurred during this time; 22 (4.4\%) deaths in the Mets group and 13 (2.5\%) deaths in the control group $(p=0.095)$. Eighteen of 35 deaths were due to cardiovascular events; 14 in the MetS group and 4 in the control group. $274(54.6 \%)$ of participants with MetS and $166(31.7 \%)$ of controls had a history of hospitalization $(p<0.0001)$. The proportion of those who had cardiovascular disease, hypertension, diabetes or other chronic diseases was statistically higher in subjects with MetS than in controls $(45.6 \%$ vs. $8.6 \%, \mathrm{p}<0.0001$, Table 2).

Table 3 shows Hazard Ratios (HRs) for all-cause and CVD mortality based on age, metabolic syndrome and its components for all participants and separately by gender in the Cox regression model. All-cause mortality was associated with age $(\mathrm{HR}=1.09)$, IFG $(\mathrm{HR}=2.53)$ and hypertension ( $\mathrm{HR}=5.53)$ in all subjects. In both sexes, all-cause mortality was associated with age (male: $H R=1.10$, fe- male: $H R=1.10$ ) and hypertension (male: $H R=5.17$, female: $\mathrm{HR}=5.96)$. CVD mortality was associated with age $(\mathrm{HR}=1.10)$, IFG $(\mathrm{HR}=3.72)$, hypertension $(\mathrm{HR}=7.60)$ and metabolic syndrome $(\mathrm{HR}=3.66)$ in all subjects. In men, CVD mortality was associated with age $(\mathrm{HR}=1.10)$ and hypertension (male: HR=8.45). In women, CVD mortality was associated with age $(\mathrm{HR}=1.10)$, IFG $(\mathrm{HR}=2.80)$ and hypertension $(\mathrm{HR}=5.96)$. The risk of all-cause and CVD mortality in all subjects was only associated with hypertension $(\mathrm{HR}=2.04,95 \% \quad \mathrm{CI}=1.01-4.11, \mathrm{p}=0.047$, and $\mathrm{HR}=2.72,95 \% \mathrm{CI}=1.01-7.37, \mathrm{p}=0.049$, respectively) after adjustment for age and gender.

Table 4 shows the HRs for the number of MetS components in association with all-cause and CVD mortality. The HRs seemed to increase as the number of components rose for both all-cause and CVD mortality. The greatest rise in CVD mortality per additional component was for five in comparison to no or only one component(s) $(\mathrm{HR}=7.55)$. This relationship did not remain significant after being controlled for age and $\operatorname{sex}(\mathrm{p}=0.78)$.

Table 3. Risk of all-cause and CVD mortality based on metabolic syndrome and its components in all participants and separately in men and women

\begin{tabular}{|c|c|c|c|c|c|c|}
\hline \multirow[t]{2}{*}{ Variable } & \multicolumn{2}{|l|}{ Men } & \multicolumn{2}{|l|}{ Women } & \multicolumn{2}{|c|}{ All subjects } \\
\hline & $\mathrm{HR}(95 \% \mathrm{CI})$ & $\mathrm{p}$ & $\mathrm{HR}(95 \% \mathrm{CI})$ & $\mathrm{p}$ & $\operatorname{HR}(95 \% \mathrm{CI})$ & $\mathrm{p}$ \\
\hline \multicolumn{7}{|c|}{ All-cause mortality } \\
\hline Age (years) & $1.10(1.06-1.12)$ & $<0.0001$ & $1.10(1.06-1.15)$ & $<0.0001$ & $1.09(1.07-1.11)$ & $<0.0001$ \\
\hline High TG & $1.71(0.48-2.83)$ & 0.73 & $0.8(0.27-2.32)$ & 0.67 & $1.003(0.51-1.97)$ & 0.990 \\
\hline Low HDL-C & $1.2(0.49-2.93)$ & 0.70 & $1.34(0.18-10.16)$ & 0.78 & $1.03(0.48-2.2)$ & 0.930 \\
\hline IFG & $2.33(0.91-5.9)$ & 0.076 & $2.8(0.99-7.9)$ & 0.051 & $2.53(1.27-5.06)$ & 0.009 \\
\hline Hypertension & $5.17(2.11-12.65)$ & $<0.0001$ & $5.96(2.12-16.77)$ & 0.001 & $5.53(2.81-10.87)$ & $<0.0001$ \\
\hline WC & $1.37(0.4-4.66)$ & 0.62 & $1.67(0.61-4.61)$ & 0.32 & $1.27(0.62-2.6)$ & 0.500 \\
\hline Metabolic syndrome & $1.6(0.66-3.87)$ & 0.29 & $2.3(0.72-7.1)$ & 0.16 & $1.75(0.88-3.47)$ & 0.110 \\
\hline \multicolumn{7}{|c|}{ Cardiovascular mortality } \\
\hline Age (years) & $1.10(1.04-1.14)$ & $<0.0001$ & $1.10(1.06-1.15)$ & $<0.0001$ & $1.10(1.07-1.15)$ & $<0.0001$ \\
\hline High TG & $2.19(0.62-7.7)$ & 0.23 & $0.80(0.27-2.32)$ & 0.52 & $1.88(0.74-4.78)$ & 0.180 \\
\hline Low HDL-C & $3.24(0.69-15.28)$ & 0.14 & $1.34(0.18-10.16)$ & 0.57 & $2.87(0.66-12.5)$ & 0.160 \\
\hline IFG & $2.10(0.52-8.4)$ & 0.29 & $2.80(0.99-7.9)$ & 0.008 & $3.72(1.43-9.65)$ & 0.007 \\
\hline Hypertension & $8.45(2.18-32.73)$ & 0.002 & $5.96(2.12-16.77)$ & 0.009 & $7.60(2.85-20.27)$ & $<0.0001$ \\
\hline WC & $1.97(0.42-9.29)$ & 0.4 & $1.67(0.61-4.61)$ & 0.58 & $1.39(0.52-3.72)$ & 0.500 \\
\hline Metabolic syndrome & $3.20(0.82-12.25)$ & 0.95 & $2.30(0.72-7.1)$ & 0.1 & $3.66(1.2-11.1)$ & 0.020 \\
\hline
\end{tabular}




\begin{tabular}{|c|c|c|c|c|}
\hline Individual components & $\begin{array}{c}\text { HR }(95 \% \mathrm{CI}) \\
\text { All-cause- mortality }\end{array}$ & $\mathrm{p}$ & $\begin{array}{l}\mathrm{HR}(95 \% \mathrm{CI}) \\
\text { CVD mortality }\end{array}$ & $\mathrm{p}$ \\
\hline 0 component (reference) & 1.00 & - & - & - \\
\hline 1 component & $2.64(0.34-20.34)$ & 0.35 & $1.00^{\mathrm{a}}$ & - \\
\hline 2 components & $5.20(0.68-39.41)$ & 0.11 & $3.36(0.98-11.52)$ & 0.053 \\
\hline 3 components & $3.57(0.46-27.45)$ & 0.22 & $2.90(0.85-9.92)$ & 0.09 \\
\hline 4 components & $3.52(0.41-30.15)$ & 0.25 & $3.92(0.98-15.67)$ & 0.053 \\
\hline 5 components & $8.90(1.04-76.10)$ & 0.046 & $7.55(1.70-33.72)$ & 0.008 \\
\hline
\end{tabular}

${ }^{\mathrm{a}} 0$ or 1 components of metabolic syndrome were considered as the reference category

\section{Discussion}

In this retrospective cohort study, we observed 35 deaths during eight years of follow-up. The hazard ratio of all-cause mortality for those with MetS was 1.75 fold (\%95CI: 0.88-3.47) higher than controls. The hazard ratio of CVD mortality in those who had MetS was 3.66 fold (\%95CI: 1.2-11.1) more than the control group although; it was not significant after being controlled for age and sex. This is in alignment with the results of the study by Wang et al. on a non-diabetic Finnish population, between 65 and 74 years old, during a 13-year follow-up period that found no association between MetS (by any definition) and all-cause mortality (14), although, a statistically significant risk for CVD mortality was observed after adjustment for confounding variables (HRs from 1.31 to 1.51). In some previous studies, including one performed in Italy no association between all-cause mortality and MetS (adjusted HR=1.24, 95\%CI: 0.60-2.59) within the elderly population was found (19). Niwa et al. did not observe a significant association between all-cause mortality and MetS after 12.5 years of follow-up in patients with metabolic syndrome in a Japanese population-based cohort study (12). Butler et al. found no significant association between all-cause mortality or CVD mortality and MetS, during a 6-year follow-up period (11). However, a number of studies reported that MetS was positively related to a higher risk of all-cause or CVD mortality (2-4, 68 ), even in Asian countries (15). Sun et al. in a cohort of 1,535 Chinese aged 50 years or older, during a 15 year follow-up period, reported that the adjusted HRs of allcause and CVD mortality in subjects who had MetS were 1.47 (95\% CI:1.20-1.80) and 1.96 (95\% CI: 1.42-2.72) higher than those without MetS, respectively (15). The conflict of results of the aforementioned study with ours might be due to a lower number of deaths $(n=35)$ and insufficient statistical power to evaluate the effect of MetS on all-cause mortality in our study. Another possible reason could be that our study was conducted in a relatively younger population.

In our study, MetS was associated with a significantly higher risk of hospital stay, CVD, DM, and HTN. Butler et al. also reported that elderly subjects with MetS were at a higher risk for coronary events (CE), myocardial infarctions (MI), heart failure (HF), and all-cause hospital stay (11). This confirms that those with MetS are at a greater risk for other chronic diseases, cardiovascular outcomes, and hospitalization.

In the present study, among the components of metabolic syndrome, hypertension and IFG were significantly associated with all-cause and CVD mortality. The associa- tion with hypertension was observed for men, women and all subjects after adjustment for age and sex while IFG was a predictor of CVD mortality in women. Our results support the findings of previous studies. A study showed that cases with both hypertension and IFG had an $82 \%$ greater mortality $(\mathrm{HR}=1.82,95 \% \mathrm{CI}: 1.58-2.09)$ in comparison with cases who had neither hypertension nor IFG (3). Zambon et al. have shown in a study on elderly Italian population that CVD mortality in women is better predicted by IFG (20). Thomas et al. reported that in participants older than 65 years, hypertension, but not metabolic syndrome, remained a strong risk factor for all-cause mortality (21). A study on a cohort of Chinese elderly adults reported a significant association between high blood pressure and CVD mortality in men $(\mathrm{HR}=3.23,95 \% \mathrm{CI}$ : 2.06 5.08) and a significant association between IFG $(\mathrm{HR}=3.68$, 95\% $\mathrm{CI}$ : 1.63-8.34), high blood pressure $(\mathrm{HR}=3.58$, 95\%CI: $1.68-7.60)$, elevated triglyceride levels $(\mathrm{HR}=0.34,95 \% \mathrm{CI}: 0.15-0.76)$ and CVD mortality in women (15). In our study, the association between MetS and CVD mortality showed a lower HR than its individual components and was not significant after being controlled for age and sex. This finding shows that the MetS does not predict CVD mortality beyond its components such as hypertension. This is in accordance with the findings of the study by Wang et al. (14).

In this study, the hazards ratios for all-cause and CVD mortality appeared to increase with the growing number of MetS components but did not persist after adjusting for age and $\operatorname{sex}(p=0.46$ and $p=0.78$, respectively). This is not consistent with previous studies. Sun et al. found a greater risk of CVD mortality for those with 3, 4, or 5 MetS components $(\mathrm{HR}=2.81-3.72)$, in comparison to subjects without any components (15). Knuiman et al. showed a progressive increase in CHD risk from one to five MetS components in Australia (22).

In this study, we also studied the association between the MetS and all-cause and CVD mortality separately in men and women. No association between all-cause or CVD mortality and the MetS was observed both in men and women. This was compatible with the findings of the study by Wang et al. that did not find an association between the MetS defined by NCEP criteria and all-cause or CVD mortality (14). However, in another study, the MetS was associated with both all-cause and CVD mortality only in men, not in women (15). This finding in our study might be due to the lack of statistical power to study the association between the MetS and all-cause or CVD mortality in men and women, separately.

Several limitations should be considered in this study. First is the small sample size, resulting in wide confidence 
intervals. Second is the number of deaths that was limited when analyses were performed separately for men and women. Third, because of the retrospective cohort study design, our study did not have sufficient information concerning confounding factors of the association between MetS and mortality. Finally, due to differences between the characteristics of subjects in two groups in our study, some of our findings might be related to these differences that have not been adjusted for.

\section{Conclusion}

The results of this study indicated that MetS was associated with a greater risk of CVD mortality, morbidity and hospital stay in this population. Among the components of metabolic syndrome, hypertension and IFG were significantly associated with all-cause and CVD mortality. However, the association of these two single components of the MetS, especially hypertension, was stronger compared to MetS as a whole. Therefore, this study confirms that MetS is a risk factor for CVD mortality, but it is not necessarily more hazardous than the risk associated with its individual components.

\section{Acknowledgments}

This study was based on an M.D. thesis (Dr. Fatemeh Karami Zarandi) and was supported by Zanjan University of Medical Sciences and Zanjan Metabolic Diseases Research Center. The authors acknowledge the Health Deputy of Zanjan University of Medical Sciences and all colleagues who were involved in the Zanjan Healthy Heart Study for their valuable support.

\section{Conflict of Interests}

The authors declare that they have no competing interests.

\section{References}

1. Forti P, Pirazzoli GL, Maltoni B, Bianchi G, Magalotti D, Muscari A, et al. Metabolic syndrome and all-cause mortality in older men and women. Eur J Clin Investig. 2012;42(9):1000-9.

2. Malik S, Wong ND, Franklin SS, Kamath TV, L'Italien GJ, Pio JR, et al. Impact of the metabolic syndrome on mortality from coronary heart disease, cardiovascular disease, and all causes in United States adults. Circulation. 2004;110(10):1245-50.

3. Mozaffarian D, Kamineni A, Prineas RJ, Siscovick DS. Metabolic syndrome and mortality in older adults: the Cardiovascular Health Study. Arch Intern Med. 2008;168(9):969-78.

4. Simons LA, Simons J, Friedlander Y, McCallum J. Does a diagnosis of the metabolic syndrome provide additional prediction of cardiovascular disease and total mortality in the elderly? The Dubbo Study. Med J Aust. 2007;186(8):400-3.

5. Wu SH, Liu Z, Ho SC. Metabolic syndrome and all-cause mortality: a meta-analysis of prospective cohort studies. Eur J Epidemiol. 2010;25(6):375-84.

6. Ford ES. The metabolic syndrome and mortality from cardiovascular disease and all-causes: findings from the National Health and Nutrition Examination Survey II Mortality Study. Atherosclerosis. 2004;173(2):309-14.

7. Isomaa B, Almgren P, Tuomi T, Forsen B, Lahti K, Nissen M, et al. Cardiovascular morbidity and mortality associated with the metabolic syndrome. Diabetes Care. 2001;24(4):683-9.

8. Kondo T, Osugi S, Shimokata K, Honjo H, Morita Y, Yamashita K, et al. Metabolic syndrome and all-cause mortality, cardiac events, and cardiovascular events: a follow-up study in 25,471 young- and middle-aged Japanese men. Eur J Cardiovasc Prev Rehabil.
2011;18(4):574-80.

9. Mottillo S, Filion KB, Genest J, Joseph L, Pilote L, Poirier P, et al. The metabolic syndrome and cardiovascular risk a systematic review and meta-analysis. J Am Coll Cardiol. 2010;56(14):1113-32.

10. Simons LA, Simons J, Friedlander Y, McCallum J. Is Prediction of Cardiovascular Disease and All-cause Mortality Genuinely Driven by the Metabolic Syndrome, and Independently from its Component Variables? The Dubbo Study. Heart Lung Circ. 2011;20(4):214-9.

11. Butler J, Rodondi N, Zhu Y, Figaro K, Fazio S, Vaughan DE, et al. Metabolic syndrome and the risk of cardiovascular disease in older adults. J Am Coll Cardiol. 2006;47(8):1595-602.

12. Niwa Y, Ishikawa S, Gotoh T, Kayaba K, Nakamura Y, Kajii E. Metabolic syndrome mortality in a population-based cohort study: Jichi Medical School (JMS) Cohort Study. J Epidemiol. 2007;17(6):203-9.

13. Noale M, Maggi S, Zanoni S, Limongi F, Zambon S, Crepaldi G. The metabolic syndrome, incidence of diabetes and mortality among the elderly: the Italian Longitudinal Study of Ageing. Diabetes Metab. 2012;38(2):135-41.

14. Wang J, Ruotsalainen S, Moilanen L, Lepisto P, Laakso M, Kuusisto J. The metabolic syndrome predicts cardiovascular mortality: a 13year follow-up study in elderly non-diabetic Finns. Eur Heart J. 2007;28(7):857-64.

15. Sun DL, Wang JH, Jiang B, Li LS, Li LS, Wu L, et al. Metabolic syndrome vs. its components for prediction of cardiovascular mortality: A cohort study in Chinese elderly adults. J Geriatr Cardiol. 2012;9(2):123-9.

16. Yen YF, Hu HY, Lin IF, Lai YJ, Su VY, Pan SW, et al. Associations of Metabolic Syndrome and its Components With Mortality in the Elderly: A Cohort Study of 73,547 Taiwanese Adults. Medicine. 2015;94(23):e956.

17. Talaei M, Sadeghi M, Marshall T, Thomas GN, Kabiri P, Hoseini S, et al. Impact of metabolic syndrome on ischemic heart disease $\& \# x 2013$; A prospective cohort study in an Iranian adult population: Isfahan cohort study. Nutr Metab Cardiovasc Dis. 2012;22(5):434-41.

18. Mellati AA, Mousavinasab SN, Sokhanvar S, Kazemi SAN, Esmailli MH, Dinmohamadi H. Correlation of anthropometric indices with common cardiovascular risk factors in an urban adult population of Iran: data from Zanjan Healthy Heart Study. Asia Pac J Clin Nutr. 2009;18(2):217-25.

19. Ravaglia G, Forti P, Maioli F, Bastagli L, Chiappelli M, Montesi F, et al. Metabolic Syndrome: prevalence and prediction of mortality in elderly individuals. Diabetes Care. 2006;29(11):2471-6.

20. Zambon S, Zanoni S, Romanato G, Corti MC, Noale M, Sartori L, et al. Metabolic syndrome and all-cause and cardiovascular mortality in an Italian elderly population: the Progetto Veneto Anziani (Pro.V.A.) Study. Diabetes Care. 2009;32(1):153-9.

21. Thomas F, Pannier B, Benetos A, Vischer UM. The impact of the metabolic syndrome--but not of hypertension--on all-cause mortality disappears in the elderly. J Hypertens. 2011;29(4):663-8.

22. Knuiman MW, Hung J, Divitini ML, Davis TM, Beilby JP. Utility of the metabolic syndrome and its components in the prediction of incident cardiovascular disease: a prospective cohort study. Eur J Cardiovasc Prev Rehabil: official journal of the European Society of Cardiology, Working Groups on Epidemiology \& Prevention and Cardiac Rehabilitation and Exercise Physiology. 2009;16(2):235-41. 